\title{
Metrics on the Moduli Spaces of Instantons Over Euclidean 4-Space
}

\author{
Antony Maciocia ${ }^{\star}$ \\ Mathematical Institute, 24-29 St. Giles, Oxford OX1 3LB, England
}

Received January 4, 1990; in revised form May 30, 1990

\begin{abstract}
We prove that the natural hyper-Kähler metrics on the moduli space of charge $k$ instantons over Euclidean four-space and on the space of ADHM matrices coincide. We use this to deduce formulae relating expressions in the curvature of a connection to invariant polynomials in the ADHM matrices corresponding to this connection. These arise from consideration of the group of symmetries acting on the moduli spaces.
\end{abstract}

\section{Introduction}

The ADHM construction of instantons identifies the moduli space of charge $k$ $S U(r)$ instantons over the 4-sphere with a space of complex matrices arising from monads. If we consider framed instantons over $\mathbb{R}^{4}$ instead, these spaces $\mathscr{M}_{k, r}$ and $\hat{\mathscr{M}}_{r, k}$ respectively, have dimension $4 r k$ and are well known to admit hyper-Kähler metrics, and it has been supposed, as a sort of folk result, that the ADHM correspondence is actually a hyper-Kähler isometry. It is one of the aims of this paper to prove this result.

Our proof boils down to identifying suitable hyper-Kähler potentials for the metrics on $\mathscr{M}_{k, r}$ and $\hat{\mathscr{M}}_{r, k}$ and proving the apparently stronger result that these potentials agree. In fact, we can view the hyper-Kähler potential on $\mathscr{M}_{k, r}$ as a potential for the dilation action of $\mathbb{R}^{4}$ lifted to the moduli space. This links our isometry with the work of Groisser and Parker $[9,10]$. As an extension of this we shall prove a general result which describes potential functions for subgroups of the conformal group acting on $\mathscr{M}_{k, r}$ in terms of the potential functions for the same groups acting on $\mathbb{R}^{4}$. We observe that the hyper-Kähler potential is also the moment map for a circle action on $\mathscr{M}_{k, r}$ (also lifted from $\mathbb{R}^{4}$ ) and deduce a formula for other moment maps of other groups of isometries of $\mathscr{M}_{k, r}$. Since we have

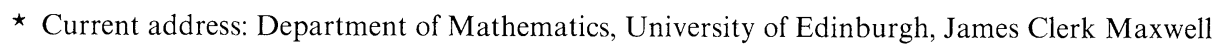
Building, King's Buildings, Mayfield Road, Edinburgh EH9 3JZ, Scotland
} 
proved that $\mathscr{M}_{k, r}$ and $\hat{\mathscr{M}}_{r, k}$ are isometric, we can immediately deduce equivalent formulae for these potential functions and moment maps in terms of the ADHM matrices.

For the sake of completeness we also provide formulae for the action of $S U(r)$ acting on $\mathscr{M}_{k, r}$ via the framing at infinity. This does not arise as the lift of an action on $\mathbb{R}^{4}$ but we can use other methods to find its moment map, see 4.14 and 4.15 .

We can view the isometry of $\mathscr{M}_{k, r}$ and $\hat{\mathscr{M}}_{r, k}$ in a more general way by extending the ADHM construction to give an equivalence of $H$-invariant instantons over $\mathbb{R}^{4}$ and $\hat{H}$-invariant instantons over $\mathbb{R}^{4 *}$. Here $H$ is a group of isometries of $\mathbb{R}^{4}$ and $\hat{H}$ is a dual group of isometries of $\mathbb{R}^{4 *}$. Again we obtain hyper-Kähler moduli spaces. In [4] Braam and van Baal prove that such spaces, where $H$ is a four dimensional lattice in $\mathbb{R}^{4}$, are isometric. The same result for $H=\mathbb{R}$ has been conjectured by Atiyah and Hitchin, see [2, chapter 16].

In fact there are several ways of proving the existence of the above isometry for the case where $H$ is a four dimensional lattice, and one expects some of these to generalize to other possible groups. One can identify the tangent spaces to the moduli spaces with suitable Ext groups and the fact that the ADHM map is an isometry can be deduced from the fact that it is functorial. This shows that the isometry arises from the formalism of the ADHM map and not from some deeper structures of the moduli spaces. It will be the aim of a future paper to describe this approach in detail.

Another way to view the agreement of the hyper-Käher potentials when $\mathbb{R}^{4} / H$ is algebraic is to observe that the potentials for more general groups $H$ are no longer just functions but norms of sections of a natural polarisation of the moduli spaces via the determinant line bundles, see [7]. Then the fact that the potentials coincide goes over to saying that the Quillen norms on the determinant lines agree. Indeed, this can be proved for the case where $H$ is a four dimensional lattice. In this way one can view Theorem 3.1 below as a degenerate version of a statement about Quillen norms on determinant line bundles or, in other words, about the spectra of twisted Laplacians acting on bundles over $\mathbb{R}^{4}$.

The paper is organised as follows. In Sect. 1 we review the ADHM construction and recall some useful formulae. The Kähler metrics on the moduli spaces are described in Sect. 2 and we prove that in the $k=1$ case these metrics agree. We also notice that the hyper-Kähler potentials extend to the Donaldson compactifications of the moduli spaces. This is used in Sect. 4 to deduce equalities of moment maps for the actions of various subgroups of the conformal group of $\mathbb{R}^{4}$ on the instanton space and the associated space of ADHM matrices. This will allow us to deduce formulae relating invariant expressions in the ADHM matrices to generalised moments of the curvature viewed as measures. This can be done by first proving directly that the two potentials agree and this is the content of Sect. 3.

\section{A Review of the ADHM Construction}

We shall review the ADHM construction in the form given in [5]. We do this partly to help the reader and partly to fix the notation and conventions to be used in subsequent sections.

We fix a principal $S U(r)$-bundle $P$ over $\mathbb{R}^{4}$ and consider the space $\mathscr{A}$ of 
connections $A$ on $P$ with $L^{2}$ integrable curvatures $F_{A}$ and which tend to a pure gauge at infinity, together with a trivialisation at infinity, we call these framed connections. Associate to $(P, A)$ the complex $r$-plane bundle-with-connection $(E, A)$ given by the standard representation of $S U(r)$ on $\mathbb{C}^{r}$. We choose the Killing form $-\operatorname{Tr}(X Y)$ on the Lie algebra $\mathfrak{s u}(r)$, and the standard Euclidean metric on $\mathbb{R}^{4}$. Then there is an action $\int\left|F_{A}\right|^{2}=-\int \operatorname{Tr} F_{A} \wedge * F_{A}$ which is conformally invariant. There is also a well defined charge $k \in \mathbb{Z}$ given by the homotopy class in $\pi_{3}(S U(r))$ of the pure gauge connection at infinity. We shall denote the space of charge $k$ connections by $\mathscr{A}_{k}$.

The gauge group $\mathscr{G}$ acts freely on $\mathscr{A}_{k}$ and the quotient $\mathscr{B}_{k}$ is a Banach manifold. We are interested in the subspace $\mathscr{M}_{k, r}$ of $\mathscr{B}_{k}$ which consists of gauge equivalence classes of framed connections which satisfy the antiself-dual (ASD) equation $F_{A}^{+}=0$, where we decompose $\Omega^{2}\left(\mathbb{R}^{4}\right)$ into its self-dual and antiself-dual parts $\Omega_{+} \oplus \Omega_{-}$. We call such connections instantons. In this case we can extend the principal bundle $P$ to the 4-sphere $S^{4}$ and use the Chern-Weil formula to compute the charge:

$$
k=-\frac{1}{8 \pi^{2}} \int \operatorname{Tr} F_{A}^{2}=-\frac{1}{8 \pi^{2}} \int\left|F_{A}^{-}\right|^{2} .
$$

The ASD equation is a first order elliptic partial differential equation and is difficult to solve. The ADHM construction makes this easier by converting the problem into one of linear algebra. We shall fix a complex structure $I$ on $\mathbb{R}^{4}$ with complex coordinates $\left(z_{1}, z_{2}\right)$. The ADHM correspondence can then be stated in the following form, see [5, Prop. 2]:

Theorem 1.1. There is a one-one correspondence between framed $S U(r)$ ASD bundles-with-connection of charge $k$ modulo gauge transformations, and matrices

$$
\left(\alpha_{1}, \alpha_{2}, a, b\right) \in M_{k \times k}(\mathbb{C}) \times M_{k \times k}(\mathbb{C}) \times M_{k \times r}(\mathbb{C}) \times M_{r \times k}(\mathbb{C})
$$

satisfying:

(i) $\left[\alpha_{1}, \alpha_{2}\right]+b a=0$,

(ii) $\left[\alpha_{1}, \alpha_{1}^{*}\right]+\left[\alpha_{2}, \alpha_{2}^{*}\right]+b b^{*}-a^{*} a=0$,

(iii) $\left(\begin{array}{c}\alpha_{1}+\lambda \\ \alpha_{2}+\lambda \\ a\end{array}\right)$ injects as a map $\mathbb{C}^{k} \rightarrow \mathbb{C}^{2 k+r}$ for all $\lambda \in \mathbb{C}$,

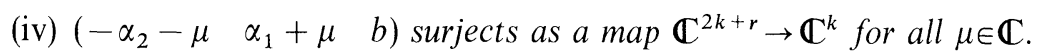

Modulo the action of $U(k)$ given by

$$
\left(\alpha_{1}, \alpha_{2}, a, b\right) \sim\left(g \alpha_{1} g^{-1}, g \alpha_{2} g^{-1}, a g^{-1}, g b\right) .
$$

We will denote this space of matrices modulo $U(k)$ by $\hat{\mathscr{M}}_{r, k}$.

If we arrange the data in the form of a monad

$$
K \stackrel{\substack{\alpha_{1} \\ \alpha_{2}}}{\longrightarrow} K \oplus K \oplus L \stackrel{\left(-\alpha_{2} \alpha_{1} b\right)}{\longrightarrow} K,
$$

where $\operatorname{dim}_{\mathbb{C}} K=k$ and $\operatorname{dim}_{\mathbb{C}} L=r$, then we can recover $E$ fibrewise via the 
cohomology of the monad, viz:

$$
E_{x} \cong \frac{\operatorname{ker}\left(\begin{array}{cc}
-\alpha_{2}+w \quad \alpha_{1}-z & b
\end{array}\right)}{\operatorname{Im}\left(\begin{array}{c}
\alpha_{1}-z \\
\alpha_{2}-w \\
a
\end{array}\right)} .
$$

The connection $A$ is given by projecting the flat connection on $\mathbb{R}^{4} \times K \oplus K \oplus L$ to $E$. The trivialisation at infinity is given by the summand $L$ in the limit $|x| \rightarrow \infty$.

Conversely $K$ can be identified with the following vector space

$$
\left\{\psi \in \Omega^{0}\left(E \otimes S^{-}\right):|\psi|=O\left(|x|^{-2}\right) \text { and } D_{A}^{-} \psi=0\right\} \text {, }
$$

where $S^{-}$is the negative spin bundle over $\mathbb{R}^{4}$ and $D_{A}^{-}$is the twisted Dirac operator. $k=1$.

We can even give explicit forms for various quantities, particularly in the case

Lemma 1.2. [8, $\mathrm{p} 104]$ If $k=1$ then there is a family of ASD connections $A$ determined by a scale $\lambda \in \mathbb{R}^{+}$and a centre $y \in \mathbb{R}^{4}$ for which

$$
\operatorname{Tr} F_{A}^{2}=\frac{48 \lambda^{4} d^{4} x}{\left(\lambda^{2}+|x-y|^{2}\right)^{4}} .
$$

Lemma 1.3. [1, chapter 2] The curvature of a connection A given by the ADHM matrices $\left(\alpha_{1}, \alpha_{2}, a, b\right)$ if $F_{A}=P d x \rho^{2} d \bar{x} P$, where $P(x)$ is the projection onto the orthogonal complement of $E_{x}$ in $K \oplus K \oplus L$ and

$$
\rho^{2}(x)=\left[\left(\alpha_{1}-z\right)^{*}\left(\alpha_{1}-z\right)+\left(\alpha_{2}-w\right)^{*}\left(\alpha_{2}-w\right)+a^{*} a\right]^{-1} \cdot \mathrm{id}_{2} .
$$

Notice that, in general, we have $\operatorname{tr} a^{*} a=\operatorname{tr} b b^{*}$ from (ii) of Theorem 1.1.

In particular, for the connections in Lemma $1,\left(\alpha_{1}, \alpha_{2}\right)$ corresponds to the centre $y$ of the instanton and the scale $\lambda^{2}$ is $a^{*} a=b b^{*}$, so that $\rho^{2}(x)=\left(|y-x|^{2}+\lambda^{2}\right)^{-1}$.

We shall now consider the Donaldson compactification $\overline{\mathscr{M}}_{k, r}$ of the moduli space $\mathscr{M}_{k, r}$. Note first that the moduli spaces we are considering are known to be connected (see [5]). It is also well known that the boundary $\partial \overline{\mathscr{M}}_{k, r}$ can be decomposed into a union of lower moduli spaces, so that

$$
\overline{\mathscr{M}}_{k, r}=\mathscr{M}_{k, r} \cup \mathbb{R}^{4} \times \mathscr{M}_{k-1} \cup S^{2} \mathbb{R}^{4} \times \mathscr{M}_{k-2} \cup \cdots \cup S^{k} \mathbb{R}^{4},
$$

where $S^{i} \mathbb{R}^{4}$ denote the $i^{\text {th }}$ symmetric product of points of $\mathbb{R}^{4}$.

Formally, we can write down the $L^{2}$ density of the curvature of an "ideal" instanton in $S^{l} \mathbb{R}^{4} \times \mathscr{M}_{k-l}$ as

$$
\left|F_{A}\right|^{2}=\left|F_{A^{\prime}}\right|^{2}+\sum_{i=1}^{l} 8 \pi^{2} \delta\left(x-y_{i}\right),
$$

where $A^{\prime} \in \mathscr{M}_{k-l}, y \in S^{l} \mathbb{R}^{4}$ are the centres of the instantons and $\delta$ denotes the Dirac delta function. The topology of $\overline{\mathscr{M}}_{k, r}$ is such that the action densities $\left|F_{A}\right|^{2}=$ $\operatorname{Tr} F_{a} \wedge F_{A}$ all converge as measures (see $[6,3.13]$ ).

There is a corresponding completion of the dual moduli space $\hat{\mathscr{M}}_{r, k}$ of ADHM matrices. This is given by $\hat{\mathscr{M}}_{r, k-l} \times S^{l} \mathbb{R}^{4} \rightarrow \overline{\hat{M}}_{r, k}$ via the maps

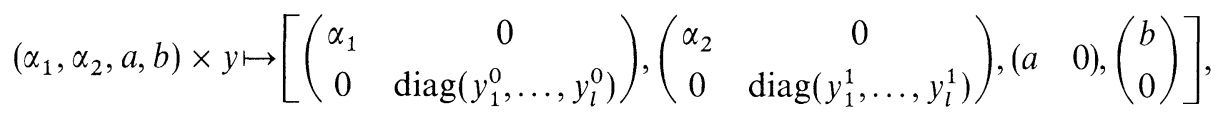


where $\left(y_{i}^{0}, y_{i}^{1}\right)$ are the complex coordinates of $y_{i} \in \mathbb{R}^{4}$. If we take the union over these inclusions one can prove that there is a one-one correspondence between $\overline{\mathscr{M}}_{k, r}$ and $\overline{\hat{M}}_{r, k}$.

\section{Metrics on the Moduli Spaces}

Recall from Taubes [14] that the $L_{1}^{2}$ tangent space to $\mathscr{M}_{k, r}$ at a gauge equivalence class of a connection in the orbifold \{unframed connections $\} / \mathscr{G}$ is isomorphic to a subspace $V_{A}$ of $L_{1}^{2} \cap \operatorname{ker}\left(d_{A}^{+} \oplus d_{A}^{*}\right) \subset \Omega^{1}(\operatorname{ad} P)$, where $d_{A}^{+}$and $d_{A}$ are the differentials in the Atiyah-Hitchin-Singer deformation complex

$$
\Omega^{\circ}(\operatorname{ad} P) \stackrel{d_{A}}{\longrightarrow} \Omega^{1}(\operatorname{ad} P) \stackrel{d_{A}^{+}}{\longrightarrow} \Omega_{+}^{2}(\operatorname{ad} P) .
$$

The remaining part of $\operatorname{ker}\left(d_{A}^{+} \oplus d_{A}^{*}\right)$ is generated by the elements

$$
d_{A} \phi_{\alpha}, \text { for } \alpha=1, \ldots, \operatorname{dim} G-h^{0},
$$

where $h^{0}$ denotes the $0^{\text {th }}$ cohomology of the AHS complex,

$$
\text { i.e. } \operatorname{ker}\left(\partial_{A}: \Omega^{0}\left(S^{4}, s^{*} \operatorname{ad} P\right) \rightarrow \Omega^{1}\left(S^{4}, s^{*} \operatorname{ad} P\right)\right) \text {. }
$$

We shall abuse notation by using $s$ to denote stereographic projection of $S^{4}$ to $\mathbb{R}^{4}$ and its "inverse." Taubes proves (in [14, Lemma 8.4]) that

$$
\operatorname{dim}\left(\operatorname{ker}\left(d_{A}^{+} \oplus d_{A}^{*}\right) \cap L_{1}^{2}\right)=\operatorname{dim}\left(\operatorname{ker}\left(d_{A}^{+} \oplus d_{A}^{*}\right) \cap L^{2}\right)=4 r k .
$$

The extra generators $d_{A} \phi_{\alpha} \in \Omega^{1}(\operatorname{ad} P)$ are determined by $\left.\sigma_{\alpha} \in s^{*} \operatorname{ad} P\right|_{\mathrm{s}(\infty)}$ and then $A+\sum \lambda_{\alpha} d_{A} \phi_{\alpha}$ is a pure gauge connection corresponding to the gauge action at infinity, and hence

Proposition 2.1. (Taubes)

$$
\begin{aligned}
T_{[A]} \mathscr{M}_{k, r} & \cong L_{1}^{2} \cap \operatorname{ker}\left(d_{A}^{+} \oplus d_{A}^{*}\right) \\
& \cong L^{2} \cap \operatorname{ker}\left(d_{A}^{+} \oplus d_{A}^{*}\right),
\end{aligned}
$$

where $\mathscr{M}$ denotes $\mathscr{M}$ framed instantons modulo gauge.

We shall now review the construction of the Kähler metrics on the instanton moduli spaces.

Fix a complex structure $I$ on $\mathbb{R}^{4}$. Let $\omega$ denote the flat Kähler form on $\mathbb{R}^{4}$ defined by $I$. This is given by $\omega(a, b)=g(I a, b)$, where $g$ is the flat Euclidean metric on $\mathbb{R}^{4}$. Then this induces a symplectic form $\Omega$ on $\mathscr{A}_{k}$ via $\Omega(u, v)=\int \operatorname{Tr}(u \wedge v) \wedge \omega / 2 \pi^{2}$, where $u, v \in T_{A} \mathscr{A}_{k}$. Here we have identified the tangent space of $\mathscr{A}$ at $A$ with the space of one forms on $\mathbb{R}^{4}$ with coefficients in the adjoint bundle ad $P$. Similarly we have an induced complex structure $\tilde{I}$ on $\mathscr{A}$. This can be done either by observing that $T \mathscr{A}$ has a natural quaternionic structure, as Taubes does, or one can proceed directly as follows

Proposition 2.2. The symplectic form $\Omega$ and complex structure $\tilde{I}$ descend to the moduli space defining an almost Kähler structure there. By almost Kähler, we mean that $\Omega(-,-)=g(\tilde{I}-,-)$ for an almost complex structure $\tilde{I}$

This is an example of the symplectic quotient construction in [11]. 
Proof. Let $\alpha \in \Omega^{1}(\operatorname{ad} P)$. Then if $d_{A}^{+} \alpha=0$ and $d_{A}^{*} \alpha=0$ the Kähler identities imply that $L^{*} d_{A} I_{\alpha}= \pm I^{-1} d_{A}^{*} I^{2} \alpha= \pm I^{-1} d_{A}^{*} \alpha=0$. Here $L^{*}$ denotes the adjoint of the operator $L \beta=\beta \wedge \omega$. Moreover $d_{A} I_{\alpha}$ has type $(1,1)$ if $d_{A} \alpha$ does. Hence $d_{A}^{+} I \alpha=0$. Similarly, $d_{A}^{*} I \alpha=I L^{*} d_{A} \alpha=0$ so that $d_{A}^{*} I \alpha=0$, and hence $\tilde{I}$ descends to $\mathscr{M}_{k, r}$.

The form $\Omega$ is well defined on $T \mathscr{M}_{k, r}$ since if $\alpha=d_{A} \gamma$, then $\Omega\left(d_{A} \gamma, \beta\right)=0$ by Stokes' theorem and the closure of $\omega$. The boundary term in $\Omega\left(d_{A} \gamma, \beta\right)$ is $\lim _{R \rightarrow \infty} \int_{S_{R}^{3}} \gamma \beta \wedge \omega$, but $\gamma \in L^{2}$ and $\beta \in L^{2}$ (strictly, we should have $\gamma \in L_{2}^{2}$ and $\beta \in L_{1}^{2}$ ). Hence $\beta \sim R^{R_{2}-\varepsilon}$ and $\gamma \sim R^{-2-\varepsilon}$, for some $\varepsilon>0$, and so the limit vanishes. It will also remain closed on $\mathscr{M}_{k, r}$ by Proposition 2.4 below and non-degenerate so that $\Omega$ defines an almost Kähler structure on $\mathscr{M}_{k, r}$ with respect to $\tilde{I}$.

In fact $\widetilde{I}$ is integrable, so that $\Omega$ is a Kähler form on $\mathscr{M}_{k, r}$. Its integrability follows by identifying $\tilde{I}$ with the natural holomorphic structure on the moduli space of holomorphic bundles over $\mathbb{C} P^{2}$ which are trivial over the line at infinity with a fixed trivialisation there, see [5].

We have Kähler potential for $\omega$ given by the function $\frac{1}{2}|x|^{2}$. By this we mean that $\omega=-i \partial \bar{\partial}|x|^{2} / 4$. We can use this to construct a Kähler potential for $\Omega$ as well.

Definition 2.3. For any connection $A$, the first and second moments $m_{1}(A)$ and $m_{2}(A)$ are defined by the formulae

$$
\begin{aligned}
& m_{1}(A)=\frac{1}{8 \pi^{2}} \int_{\mathbb{R}^{4}} x . \operatorname{Tr} F_{A}^{2} \in \mathbb{R}^{4}, \\
& m_{2}(A)=\frac{1}{16 \pi^{2}} \int_{\mathbb{R}^{4}}|x|^{2} \operatorname{Tr} F_{A}^{2} \in \mathbb{R} .
\end{aligned}
$$

These descend to the moduli spaces $\mathscr{M}_{k, r}$.

Proposition 2.4. The second moment is a Kähler potential for the Kähler form $\Omega$ on $\mathscr{M}_{k, r}$

Proof. Endow $E$ with a holomorphic structure compatible with $A$. Pick $\alpha, \bar{\alpha} \in T_{[A]} \mathscr{M}_{k, r} \otimes \mathbb{C}$ so that $\bar{\partial}_{A} \alpha=0=\partial_{A} \bar{\alpha}$. Note that, if $t>0$ is real, then $F_{A+t a}^{2}=F_{A}^{2}+2 t F_{A} \wedge d_{A} \alpha+O\left(t^{2}\right)$ and hence

$$
\begin{aligned}
-\frac{1}{2} i \frac{\partial}{\partial \bar{\alpha}} \frac{\partial}{\partial \alpha} m_{2} & =\frac{1}{4 \pi^{2}} \frac{\partial}{\partial \bar{\alpha}} \int \frac{-i}{4}|x|^{2} \operatorname{Tr} F_{A} \wedge \partial_{A} \alpha \\
& =\frac{1}{2 \pi^{2}} \int \frac{-i}{4}|x|^{2} \operatorname{Tr}\left[\left(\bar{\partial}_{A} \bar{\alpha} \wedge \partial_{A} \alpha\right)+F_{A} \wedge \alpha \wedge \bar{\alpha}\right] .
\end{aligned}
$$

Recall also that $F_{A} \wedge \alpha \wedge \bar{\alpha}=\bar{\partial}_{A} \partial_{A} \alpha \wedge \bar{\alpha}$ and so, using Stokes' theorem twice, we have

$$
-\frac{1}{2} i \frac{\partial}{\partial \bar{\alpha}} \frac{\partial}{\partial \alpha} m_{2}=\frac{1}{2 \pi^{2}} \int \omega \wedge \operatorname{Tr}(\alpha \wedge \bar{\alpha}) .
$$

Hence $-\frac{1}{2} i \bar{\partial} \partial m_{2}(\alpha, \beta)=\Omega(\alpha, \beta)$.

Remark. The above result generalises to arbitrary Kähler manifolds $X$ with Kähler potentials $f$ to say that there is a Kähler potential proportional to $\int_{X} f \operatorname{Tr} F_{A}^{2}$ for the natural $L^{2}$ Kähler metric on the moduli spaces of anti-selfdual connections on bundles over $X$, at least for those $X$ for which Stokes' theorem still gives the 
desired conclusion in the above proof, e.g. all compact Kähler varieties. Typically, $f=\log \|s\|^{2}$, where $s$ is a nontrivial section of a polarisation of $X$, see [7].

We can extend this to a hyper-Kähler structure on $\mathscr{M}_{k, r}$ using the natural hyper-Kähler structure on $\mathbb{R}^{4}$ given by symplectic forms $\omega=\omega_{1}, \omega_{2}, \omega_{3}$ and complex structures $I=I_{1}, I_{2}, I_{3}$. These all define forms $\Omega_{i}$ and complex structures $\widetilde{I}_{i}$ on $\mathscr{M}_{k, r}$, and, by Proposition 2.4 , form a hyper-Kähler structure on $\mathscr{M}_{k, r}$.

Combining the above results we have proved:

Theorem 2.5. The moduli space $\mathscr{M}_{k, r}$ is a hyper-Kähler manifold of dimension $4 r k$ with complex structures $I_{1}, I_{2}, I_{3}$ inherited from $\Omega^{*}\left(\mathbb{R}^{4}\right)$ and Kähler forms $\Omega_{1}, \Omega_{2}$, $\Omega_{3}$, where $\Omega_{i}=\int \operatorname{Tr}(u \wedge v) \wedge \omega_{i} / 2 \pi^{2}$. Moreover $\mathscr{M}_{k, r}$ admits $m_{2}$ as a hyper-Kähler potential.

This can also be viewed in terms of the hyper-Kähler quotients in [11]

The hyper-Kähler structure on $\hat{\mathscr{M}}_{r, k}$ is simpler since the flat Kähler structure on the space of matrices $M_{k \times k}(\mathbb{C}) \times M_{k \times k}(\mathbb{C}) \times M_{r \times k}(\mathbb{C}) \times M_{k \times r}(\mathbb{C})$ is invariant under the $U(k)$ action given in Sect. 1. For different choices of complex structure $I$ on $\mathbb{R}^{4}$ we obtain isomorphic spaces $\hat{\mathscr{M}}_{r, k}(I)$, so we can identify these but with different complex structures $\hat{I}$. In particular we obtain a hyper-Kähler structure $\hat{\Omega}_{j}, \hat{I}_{j}$ on $\hat{\mathscr{M}}_{r, k}$ when we take the usual Marsden-Weinstein quotient $\mu^{-1}(0) / U(k)$, where $\mu$ is the moment map for the action of $U(k)$. This is done in [3] where the hyper-Kähler structure on $\mathscr{M}_{k, r}$ is induced by pullback from $\hat{\mathscr{M}}_{r, k}$. We shall prove in the following lemma and in the next section that this hyper-Kähler structure coincides with the one given in Theorem 2.5 .

We note in passing that the holomorphic interpretation of $\mathscr{M}_{k, r}$ shows that the ADHM map preserves the complex structures and so the equivalence of the Kähler structures will imply that the moduli spaces $\mathscr{M}_{k, r}$ and $\hat{\mathscr{M}}_{r, k}$ are isometric as Riemannian manifolds.

There is an obvious Kähler potential for these Kähler forms given by the norm squared of the matrices, viz $\frac{1}{2}\left(\left\|\alpha_{1}\right\|^{2}+\left\|\alpha_{2}\right\|^{2}+\|a\|^{2}+\|b\|^{2}\right)$.

In the next section we shall prove the general result

$$
\frac{1}{2}\|p(A)\|^{2}=m_{2}(A),
$$

where we shall use $p$ to denote the ADHM correspondence. In the case $k=1$ the direct computation is easy:

Lemma 2.6. If $A$ is a $k=1$ instanton as in Lemma 1.2 with scale $\lambda$ and centre $y$, then $\frac{1}{2}\|p(A)\|^{2}=m_{2}(A)$. Hence $\mathscr{M}_{1, r}$ is isometrically isomorphic to $\hat{\mathscr{M}}_{r, 1}$ under $p$.

Proof. From 1.2 we have a formula for $\operatorname{Tr} F_{A}^{2}$, so we can compute both sides of (*) explicitly.

$$
\begin{aligned}
2 m_{2}(A) & =\frac{6}{\pi^{2}} \lambda^{4} \int_{\mathbb{R}^{4}} \frac{|x+y|^{2} d^{4} x}{\left(\lambda^{2}+|x|^{2}\right)^{4}} \\
& =\frac{6}{\pi^{2}} \lambda^{4} \int_{\mathbb{R}^{4}} \frac{|x|^{2}+|y|^{2} d^{4} x}{\left(\lambda^{2}+|x|^{2}\right)^{4}} \\
& =\frac{6}{\pi^{2}}\left(2 \lambda^{2}+|y|^{2}\right) \cdot \frac{\pi^{2}}{6} \\
& =\|p(A)\|^{2} .
\end{aligned}
$$


The last line follows since $\|p(A)\|^{2}=|y|^{2}+a^{*} a+b b^{*}=|y|^{2}+2 \lambda^{2}$.

There is a corresponding result for the first moment of a connection:

Lemma 2.7. For a connection $A$ as above, $m_{1}(A)=\left(\operatorname{tr} \alpha_{1}, \operatorname{tr} \alpha_{2}\right) \in \mathbb{C}^{2}$, where $p(A)=\left(\alpha_{1}, \alpha_{2}, a, b\right)$.

Proof. When $k=1$ the right-hand side is just $y$, the centre. The first moment can be explicitly computed as above to give $y$, as desired.

We shall use Lemma 2.7 as the first step in an induction proof that the formula $m_{1}(A)=\left(\operatorname{tr} \alpha_{1}, \operatorname{tr} \alpha_{2}\right)$ holds for all values of $k$.

\section{On a Formula of Osborn}

In principle, we could evaluate $\int|x|^{2} \operatorname{Tr} F_{A}^{2}$ directly, as in Lemma 2.6, for general values of $k$ to verify the formula $m_{2}(A)=\frac{1}{2}\|p(A)\|^{2}$ without any assumptions about the metrics (and hence prove that the metrics coincide), but in practice this is impossible. Our only hope would be to integrate by parts and for this we would like to express $\operatorname{Tr} F_{A}^{2}$ as a total differential. Fortunately there is a formula of Osborn $[12,5.5]$ which does just this. In our conventions the formula reads

$$
* \operatorname{Tr} F_{A}^{2}=-\frac{1}{2} \Delta \Delta \log \operatorname{det} L(x),
$$

where $L(x)=\left(\alpha_{1}-z_{1}\right)^{*}\left(\alpha_{1}-z_{1}\right)+\left(\alpha_{2}-z_{2}\right)^{*}\left(\alpha_{2}-z_{2}\right)+a^{*} a$ for $\left(\alpha_{1}, \alpha_{2}, a, b\right)=p(A)$, and $\Delta$ is the Euclidean Laplacian on $\mathbb{R}^{4}$.

There is an obvious interpretation of $L(x)$ as a family of $\mathbb{R}^{4}$ indexed Laplacian operators on the "Dolbeault complex" $K \rightarrow K \oplus K \oplus L \rightarrow K$ in degree 0 . Since this complex is a monad for all $x \in \mathbb{R}^{4}, L(x)$ must be invertible for all $x$. Osborn's proof of this can be viewed in this way and the evaluation follows certain renormalisations interpreted in this linear algebraic context. This is the best way of understanding the formula but it can, however, be verified directly from the formulae of Sect. 1. In particular, it is easy to verify for $k=1$ instantons directly.

The derivatives of $\log \operatorname{det} L$ are:

$$
\begin{aligned}
d_{i} \log \operatorname{det} L= & \operatorname{tr} M L_{i}^{\prime}, \\
\Delta \log \operatorname{det} L= & 8 \operatorname{tr} M-\sum_{i} \operatorname{tr}\left(M L_{i}^{\prime} M L_{i}^{\prime}\right), \\
d_{j} \Delta \log \operatorname{det} L= & -12 \operatorname{tr} M^{2} L_{j}^{\prime}-2 \sum_{i} \operatorname{tr}\left(M L_{j}^{\prime} M L_{i}^{\prime} M L_{i}^{\prime}\right) \\
-\frac{1}{2} \Delta \Delta \log \operatorname{det} L= & \operatorname{tr}\left[48 M^{2}-\sum_{j} 24 M^{2} L_{j}^{\prime} M L_{j}^{\prime}\right. \\
& \left.+\sum_{i, j}\left(2 M L_{j}^{\prime} M L_{j}^{\prime} M L_{i}^{\prime} M L_{i}^{\prime}+M L_{j}^{\prime} M L_{i}^{\prime} M L_{j}^{\prime} M L_{i}^{\prime}\right)\right],
\end{aligned}
$$

where $M=L^{-1}$. On the other hand the formula of Atiyah given in Lemma 1.2 can be used to directly compute $\operatorname{Tr} F_{A}^{2}$ :

$* \operatorname{Tr} F_{A}^{2}=-16 \operatorname{tr}\left\{\left[A_{1} M, A_{2}^{*} M\right]\left[A_{1}^{*} M, A_{2} M\right]+3\left(A_{1} M A_{1} M+A_{2} M A_{2} M-M\right)^{2}\right\}$, where $A_{i}=\alpha_{i}-z_{i}$ and the brackets denote the commutator $[B, C]=B C-C B$. If we now substitute for $L_{i}$ in the formula for $\Delta^{2} \log \operatorname{det} L$ we obtain the above formula for $* \operatorname{Tr} F_{A}^{2}$. Thus proving Osborn's formula. 
We can now integrate

$$
m_{2}(A)=\frac{1}{32 \pi^{2}} \int|x|^{2} \Delta^{2} \log \operatorname{det} L(x) d^{4} x
$$

by parts, to obtain

$$
m_{2}(A)=\frac{1}{32 \pi^{2}} \lim _{R \rightarrow \infty} \int_{S_{R}^{3}}\left[R^{4} x . d \Delta \log \operatorname{det} L-2 R^{4} \Delta \log \operatorname{det} L+8 R^{2} x \cdot d \log \operatorname{det} L\right] d S,
$$

where $R^{3} d S$ is the measure on $S_{R}^{3}$.

It is convenient to introduce the vector of matrices $\gamma$ where

$$
\begin{aligned}
& \gamma_{1}=\frac{1}{2}\left(\alpha_{1}+\alpha_{1}^{*}\right), \quad \gamma_{2}=\frac{i}{2}\left(\alpha_{1}-\alpha_{1}^{*}\right), \\
& \gamma_{3}=\frac{1}{2}\left(\alpha_{2}+\alpha_{2}^{*}\right), \quad \gamma_{4}=\frac{i}{2}\left(\alpha_{2}-\alpha_{2}^{*}\right),
\end{aligned}
$$

Then the derivatives of $L$ are $L_{i}^{\prime}=2\left(x_{i}-\gamma_{i}\right)$. If we substitute these and the derivatives of $\log \operatorname{det} L$ into the integrand of $(*)$ we obtain

$$
\begin{aligned}
& \operatorname{tr}\left[16 R^{2}\langle\gamma, x\rangle L^{2} M^{3}-24 R^{4}\left(R^{2}-\langle\gamma, x\rangle\right) L M^{3}\right. \\
& \left.\quad+2 R^{4}\left(L+2 R^{2}-2\langle\gamma, x\rangle\right)\left(R^{2} M^{2}+\langle M \gamma, M \gamma\rangle-2\langle\gamma, x\rangle M^{2}\right) M\right] d S .
\end{aligned}
$$

Here we must take care with non-commuting matrices, but we can cyclically commute using the properties of trace.

In the limit as $R$ tends to infinity the matrix $M$ tends to $R^{2} . I_{k}$, where $I_{k}$ denotes the $k \times k$ identity matrix. Hence we can neglect terms which have order smaller than 1. Now substitute for $L=\alpha_{1}^{*} \alpha_{1}+\alpha_{2}^{*} \alpha_{2}+R^{2}-2\langle\gamma, x\rangle+a^{*} a$ in the integrand to obtain

$$
\begin{aligned}
& \operatorname{tr}\left[\left(-16 R^{6}\left(\alpha_{1}^{*} \alpha_{1}+\alpha_{2}^{*} \alpha_{2}+a^{*} a\right)-24 R^{6}\langle\gamma, x\rangle\right.\right. \\
& \left.\left.\quad+80 R^{4}\langle\gamma, x\rangle^{2}\right) M^{3}+24 R^{6}\langle M \gamma, M \gamma\rangle M\right] d S .
\end{aligned}
$$

The contribution from the last term in the large $R$ limit is $48 \pi^{2}\left(\left\|\alpha_{1}\right\|^{2}+\left\|\alpha_{2}\right\|^{2}\right)$. From the $x \mapsto-x$ symmetry the potentially divergent term $R^{6}\langle\gamma, x\rangle$ can be computed as follows:

$$
\begin{aligned}
\int_{S_{R}^{3}} \operatorname{tr} R^{6}\langle\gamma, x\rangle M^{3} d S & =\frac{1}{2} \int_{S_{R}^{3}} \operatorname{tr} R^{6}\langle\gamma, x\rangle\left(L(-x)^{3}-L(x)^{3}\right) M(x)^{3} M(-x)^{3} d S \\
& =6 \int_{S_{R}^{3}} \operatorname{tr} R^{10}\langle\gamma, x\rangle^{2} M(x)^{3} M(-x)^{3} d S \\
& \simeq 6 \int_{S_{R}^{3}} \operatorname{tr} \frac{\langle\gamma, x\rangle^{2}}{R^{2}} d S \text { in the large } R \text { limit } \\
& =6 \int_{S_{R}^{3}} \operatorname{tr}\left[\frac{\alpha_{1}^{*} \alpha_{1}}{2} \cdot \frac{\left(x_{1}^{2}+x_{2}^{2}\right)}{R^{2}}+\frac{\alpha_{2}^{*} \alpha_{2}}{2} \cdot \frac{\left(x_{3}^{2}+x_{4}^{2}\right)}{R^{2}}\right] d S \\
& =3 \pi^{2}\left(\left\|\alpha_{1}\right\|^{2}+\left\|\alpha_{2}\right\|^{2}\right) .
\end{aligned}
$$


Hence we finally obtain

$$
\begin{aligned}
m_{2}(A)= & -\frac{1}{32 \pi^{2}}\left[-32 \pi^{2}\left(\left\|\alpha_{1}\right\|^{2}+\left\|\alpha_{2}\right\|^{2}+\|a\|^{2}\right)\right. \\
& \left.+(-72+40+48) \pi^{2}\left(\left\|\alpha_{1}\right\|^{2}+\left\|\alpha_{2}\right\|^{2}\right)\right] \\
= & \frac{1}{2}\left(\left\|\alpha_{1}\right\|^{2}+\left\|\alpha_{2}\right\|^{2}+\|a\|^{2}+\|b\|^{2}\right) .
\end{aligned}
$$

Thus we have proved:

Theorem 3.1. For any $[A] \in \mathscr{M}_{k, r}, m_{2}(A)=\frac{1}{2}\|p(A)\|^{2}$.

This, together with Theorem 2.5, implies:

Theorem 3.2. The ADHM correspondence is a hyper-Kähler isometry of $\mathscr{M}_{k, r}$ and $\mathscr{M}_{r, k}$.

\section{Homothetic Isometries and Moment Maps}

We start by recalling a result of Groisser and Parker [9, Eq. 4.3] which can be stated in the following form.

Proposition 4.1. Let $H$ be a subgroup of the conformal group of a four-manifold $M$ with a lift of its action to a principal bundle $P \rightarrow M$. Let the infinitesimal action be given by $X_{h}$ for $h \in \mathfrak{H}(=\operatorname{Lie}(H))$. Then the infinitesimal action of $H$ on $\mathscr{A}_{k}$ is given by

$$
\left.Y_{h}(A)=X_{h}\right\lrcorner F_{A}+d_{A} u,
$$

where $u \in \Omega^{0}(\operatorname{ad} P)$ represents $\left.X_{h}\right\lrcorner \omega_{A} \in \Omega^{0}(P ; \mathfrak{g})$, and $\omega_{A}$ is the connection form.

In [9] they use the example of $P \rightarrow S^{4}$ which is the $S U(2)$ bundle which double covers the bundle of orthonormal frames in $\Lambda_{+} T^{*} S^{4}$ and so comes with a lift of the conformal group action on $S^{4}$.

Definition 4.2. If $H$ is a group acting on a Riemannian manifold $M$, then call the action exact if $X_{h}=-\nabla f_{h}$ for some $f \in \Omega^{0}\left(\mathfrak{S}^{*}\right)$.

An important example of these is the subgroup of the group of homothetic isometries of Euclidean 4-space given by the semidirect product $H=\mathbb{R}^{4} \rtimes \mathbb{R}^{+}$. The action of $(y, \lambda) \in H$ is the linear one $x \mapsto \lambda x+y$. We shall be interested in the groups $\mathbb{R}^{4}$ and $\mathbb{R}^{+}$separately. These are exact with $f_{\lambda}=\lambda\|x\|^{2} / 2$ and $f_{y}=(x, y)$. We shall use $Y_{i}, i=1,2$, to denote the separated actions on the space of connections. It is easy to see that these are all the possible exact conformal actions on $\mathbb{R}^{4}$.

We can regard the two moments $m_{1}$ and $m_{2}$ defined in Sect. 2 as taking values in the dual of the Lie algebras of $\mathbb{R}^{4}$ and $\mathbb{R}^{+}$. We would like to prove that the actions $Y_{i}$ descend to $\mathscr{M}_{k, \boldsymbol{r}}$ and that $-\nabla m_{i}=Y_{i}$.

Theorem 4.3. If $H$ is a subgroup of $\mathbb{R}^{4} \times \mathbb{R}^{+}$acting on $\mathbb{R}^{4}$, then the induced action on $\mathscr{A}_{k}$ descends to $\mathscr{M}_{k, r}$. Moreover, this action is exact with associated function

$$
m(A)=\frac{1}{8 \pi^{2}} \int_{\mathbb{R}^{4}} f \operatorname{Tr} F_{A}^{2} .
$$

Proof. To show that $Y_{h}$ descends to $\mathscr{M}_{k, r}$ it suffices to prove that $d_{A}^{+} Y=0$. Now 
$d_{A}^{+} d_{A}=0$ as $A$ is ASD. So

$$
\begin{aligned}
d_{A}^{+} Y & \left.=d_{A}^{+}(X\lrcorner F_{A}\right) \\
& \left.=P_{+} d_{A}(X\lrcorner F_{A}\right) \\
& =H^{0} f \cdot F_{A} \quad \text { see }[10, \text { Lemma 3.1]. }
\end{aligned}
$$

Here $H^{0}$ denotes the trace-free part of the Hessian. For the group $H$ of the theorem this vanishes. This is because $H$ is conformally Killing so that the Hessian of $f$ is proportional to the metric which has no trace-free part.

Noting the identity $\uparrow \alpha\lrcorner * \beta=*(\alpha \wedge \beta)$ for a 1 -form $\alpha$ and 2 -form $\beta$, we have

$$
\begin{aligned}
\delta_{A} m(b) & =\frac{1}{8 \pi^{2}} \int f \operatorname{Tr}\left(d_{A} b \wedge F_{A}\right) \\
& =-\frac{1}{4 \pi^{2}} \int d f \wedge \operatorname{Tr}\left(b \wedge F_{A}\right) \\
& \left.=-\frac{1}{4 \pi^{2}} \int \operatorname{Tr}\left[(\uparrow d f\lrcorner * F_{A}\right) \wedge * b\right] \\
& \left.=-\frac{1}{4 \pi^{2}} \int \operatorname{Tr}\left[(X\lrcorner F_{A}\right) \wedge * b\right] \\
& \left.=-\langle X\lrcorner F_{A}, b\right\rangle \\
& =-Y(A)(b) .
\end{aligned}
$$

We can justify the use of Stokes' theorem in the first line as the integral $\int_{S^{3}} f \operatorname{Tr}\left(b \wedge F_{A}\right)$ over large spheres $S_{R}^{3}$ vanish in the limit $R \rightarrow \infty$, as $f b F_{A}=$ $S_{R}^{3}\left(R^{-4}\right)$.

The notion of exact action is similar to that of Hamiltonian action when the action is symplectic. The associated function is then the moment map. We can prove an analogous result to the one above in this context too.

Theorem 4.4. Let $H$ be a subgroup of the conformal group of $\mathbb{R}^{4}$ acting symplectically on $\mathbb{R}^{4}$ such that the action is Hamiltonian with moment map $\mu$. Suppose further that $\nabla I \nabla \mu$ has skew trace-free part. Then the action defined above descends to $\mathscr{M}_{k, r}$ and is Hamiltonian there with moment map

$$
\tilde{\mu}(A)=\frac{1}{8 \pi^{2}} \int \mu \operatorname{Tr} F_{A}^{2},
$$

provided $\mu=O\left(|x|^{2}\right)$ as $|x| \rightarrow \infty$.

Proof. Observe that $X_{h}=-I \nabla \mu_{h}$ generates the $\mathfrak{H}$ action. Then replacing $H f$ in the proof of Theorem 4.3 with $\nabla I \nabla \mu$ implies that the action descends to $\mathscr{M}_{k, r}$. To prove that we have the correct moment map note that

$$
\begin{aligned}
\delta_{A} \tilde{\mu}(b) & =\frac{1}{4 \pi^{2}} \int \mu \operatorname{Tr}\left(d_{A} b \wedge F_{A}\right) \\
& \left.\left.=\frac{1}{4 \pi^{2}} \int \operatorname{Tr}\left[((X\lrcorner \omega) \wedge F_{A}\right) \wedge b\right)\right]
\end{aligned}
$$




$$
\begin{aligned}
& \left.=-\frac{1}{2 \pi^{2}} \int \operatorname{Tr}\left[(X\lrcorner F_{A}\right) \wedge b\right] \wedge \omega \quad \text { since } F_{A} \wedge \omega=0 \\
& \left.=-\Omega(X\lrcorner F_{A}, b\right) . \quad \square
\end{aligned}
$$

Recalling that the measures $\operatorname{Tr} F_{A}^{2}$ converge on $\bar{M}_{k, r}$ then the integrals in the above theorems will extend to continuous maps on $\overline{\mathscr{M}}_{k, r}$ and hence:

Corollary 4.5. The formulae of Theorems 4.3 and 4.4 hold on $T \overline{\mathscr{M}}_{k, r}$.

We can now deduce the main theorem of this section:

Theorem 4.6. Let $H$ be any group acting on $\mathbb{R}^{4}$ whose action is exact (respectively, Hamiltonian), with associated maps on $\mathscr{M}_{k, r}$ and $\hat{\mathscr{M}}_{r, k}$ given by $m(A)$ and $\hat{m}(M)$ (respectively, $\mu(A)$ and $\hat{\mu}(M)$ ). Suppose further that $m(A)=\hat{m}(p(A))$ on $\mathscr{M}_{k, r}$ (respectively, $\mu(A)=\hat{\mu}(p(A)))$, for $k=1$. Then these equalities hold for all values of $k$.

Proof. Since $Y=p^{*} \hat{Y}$, where $Y$ and $\hat{Y}$ are infinitesimal generators of the $H$ action on $\mathscr{M}_{k, r}$ and $\hat{\mathscr{M}}_{r, k}$ respectively, and from the definition of moment maps and associated functions, there is a constant $\kappa \in \mathbb{R}^{+}$such that $\nabla \phi_{\kappa}=0$, where $\phi_{\kappa}(A)=\kappa m(A)-\hat{m}(p(A))$, or $\phi_{\kappa}(A)=\kappa \mu(A)-\hat{\mu}(p(A))$ for $[A] \in \overline{\mathscr{M}}_{k, r}$, depending on whether the action of our group is exact or Hamiltonian. Observe that $\mathscr{M}_{k, r}$ are all connected and that $S^{k-1} \mathbb{R}^{4} \times \mathscr{M}_{1, r} \subset \overline{\mathscr{M}}_{k, r}$. Then the hypothesis of the theorem shows that $\phi_{\kappa} \equiv 0$ and $\kappa=1$.

We now apply the above to the homothetic actions on $\hat{\mathscr{M}}_{r, k}$. These are given by $\left(\alpha_{1}, \alpha_{2}, a, b\right) \mapsto\left(\lambda \alpha_{1}+z_{1}, \lambda \alpha_{2}+z_{2}, \lambda a, \lambda b\right)$ and we can complexify the action to that of $\mathbb{C}^{2} \times \mathbb{C}^{*}$ via the complex structure on $\mathbb{R}^{4}$.

Lemma 4.7. The above actions are exact with associated functions

$$
\begin{gathered}
\hat{f}_{1}\left(\alpha_{1}, \alpha_{2}, a, b\right)=\left(\operatorname{tr} \alpha_{1}, \operatorname{tr} \alpha_{2}\right), \\
\hat{f}_{2}\left(\alpha_{1}, \alpha_{2}\right)=\frac{1}{2}\left(\left\|\alpha_{1}\right\|^{2}+\left\|\alpha_{2}\right\|^{2}+\|a\|^{2}+\|b\|^{2}\right) .
\end{gathered}
$$

Proof.

while

$$
\frac{1}{2} d\left(\left\|\alpha_{1}\right\|^{2}+\left\|\alpha_{2}\right\|^{2}+\|a\|^{2}+\|b\|^{2}\right)=\alpha_{1} d \alpha_{1}+\alpha_{2} d \alpha_{2}+a d a+b d b,
$$

$$
d\left(\operatorname{tr} \alpha_{1}, \operatorname{tr} \alpha_{2}\right)\left(y_{1}, y_{2}\right)=y_{1} \operatorname{tr} d \alpha_{1}+y_{2} \operatorname{tr} d \alpha_{2}
$$

It is easy to see that these equal $\uparrow \hat{X}_{1}$ and $\uparrow \hat{X}_{2}$ respectively.

Recalling Lemma 2.7 we have:

Corollary 4.8. For all $k$ and $[A] \in \mathscr{M}_{k, r}$, set $\left(\alpha_{1}, \alpha_{2}, a, b\right)=p(A)$. Then

$$
m_{1}(A)=\left(\operatorname{tr} \alpha_{1}, \operatorname{tr} \alpha_{2}\right) .
$$

Remark 4.9. Applying this to the dilation action we see that the moduli spaces $\mathscr{M}_{k, r}$ and $\mathscr{M}_{r, k}$ are isometric if and only if their Kähler potentials agree.

Consider the $S^{1}$ action on $\mathbb{R}^{4}$ which permutes $I_{2}, I_{3}$. A moment map for this is $|x|^{2} / 2$. This is because $\nabla|x|^{2}$ generates dilations and hence $I_{1} \nabla|x|^{2}$ generates rotations permuting $I_{2}$ and $I_{3}$, but fixing $I_{1}$. This is the construction of the hyper-Kähler potential given in [11, pages 550-554]. Indeed this implies the existence of a hyper-Kähler potential on $\mathscr{M}_{k, r}$ as in Theorem 2.5 and provides us with an alternative proof of that theorem. Hence we have proved 
Proposition 4.10. The second moment $m_{2}$ is a moment map for each of the complex structures $I_{i}$ with respect to the $S^{1}$ action which fixes $I_{i}$ and permutes the other two complex structures.

These $S^{1}$ 's generate the nonsymplectic $S O(3)$ action.

Corollary 4.11. The manifold $m_{2}^{-1}(x) / S O(3) \subset \mathscr{M}_{k, r} / S O(3)$, for any nondegenerate point $x \in \operatorname{Im} m_{2}$, is a quaternionic Kähler manifold for each $k$ and $r$.

This provides us with a large selection of quaternionic Kähler manifolds.

If we now turn to the other $S O(3)$ in the rotation group $S O(4)$ of $\mathbb{R}^{4}$, we see that this action is given, on the Lie algebra level, by $I, J, K$. These cover the actions on $\mathbb{R}^{4}$ given by (counter) rotations of pairs of $\mathbb{C} \subset \mathbb{R}^{4}$. The respective moment maps are

$$
\begin{cases}x_{1} x_{2}+x_{3} x_{4} & \text { for the complex structure } K \\ x_{1} x_{3}+x_{2} x_{4} & \text { for the complex structure } I \\ x_{1} x_{4}+x_{2} x_{3} & \text { for the complex structure } J\end{cases}
$$

The matrices $\nabla X$ for generators $X$ of these actions are skew symmetric and hence satisfy the conditions of Theorem 4.4 .

The action on the ADHM matrices is also by rotation on $\left(\alpha_{1}, \alpha_{2}\right)$, so the moment maps are given by $\operatorname{Re}\left\langle M\left(\alpha_{1}, \alpha_{2}\right),\left(\alpha_{1}, \alpha_{2}\right)\right\rangle$, where $M=\hat{I}, \hat{J}$ or $\hat{K}$ (for example $\left.\hat{I}=\operatorname{diag}\left(\left(\begin{array}{ll}0 & 1 \\ 1 & 0\end{array}\right),\left(\begin{array}{ll}0 & 1 \\ 1 & 0\end{array}\right)\right)\right)$. If we compute these we find moment maps (up to scalar multiples of $2^{m}$ )

$$
\begin{cases}\operatorname{Im} \operatorname{tr}\left(\alpha_{1} \alpha_{1}^{t}+\alpha_{2} \alpha_{2}^{t}\right) & \text { for the complex structure } K \\ \operatorname{Re} \operatorname{tr}\left(\alpha_{1} \alpha_{2}^{*}\right) & \text { for the complex structure } I \\ \operatorname{Im} \operatorname{tr}\left(\alpha_{1} \alpha_{2}^{t}\right) & \text { for the complex structure } J\end{cases}
$$

where $t$ denotes transpose of the matrix. Notice that the moment map corresponding to the complex structure $I$ is distinguished. This is because the matrices themselves arose from fixing the complex structure $I$ on $\mathbb{R}^{4}$.

It is easy to see that $\int\left(x_{1} x_{3}+x_{2} x_{4}\right) \operatorname{Tr} F_{A}^{2} / 8 \pi^{2}=\operatorname{Re} \operatorname{tr} \alpha_{1} \alpha_{2}^{*}$ for the $k=1$ case, since

$$
\int \frac{\left(x_{1} x_{3}+x_{2} x_{4}\right) d^{4} x}{\left(\lambda^{2}+|x-y|^{2}\right)^{4}}=\int \frac{\left(y_{1} y_{3}+y_{2} y_{4}\right) d^{4} x}{\left(\lambda^{2}+|x|^{2}\right)^{4}} .
$$

Hence, by Theorem 4.4, this equality holds for all $k$. The other cases follow similarly, and so we have:

Theorem 4.12. For all $k, r$, let $\left(\alpha_{1}, \alpha_{2}, a, b\right)=p(A)$ for $A \in \mathscr{M}_{k, r}$. Then

$$
\begin{aligned}
& \frac{1}{8 \pi^{2}} \int\left(x_{1} x_{3}+x_{2} x_{4}\right) \operatorname{Tr} F_{A}^{2}=\operatorname{Re} \operatorname{tr}\left(\alpha_{1} \alpha_{2}^{*}\right), \\
& \frac{1}{4 \pi^{2}} \int\left(x_{1} x_{2}+x_{3} x_{4}\right) \operatorname{Tr} F_{A}^{2}=\operatorname{Im} \operatorname{tr}\left(\alpha_{1} \alpha_{1}^{t}+\alpha_{2} \alpha_{2}^{t}\right),
\end{aligned}
$$


and

$$
\frac{1}{8 \pi^{2}} \int\left(x_{1} x_{4}+x_{2} x_{3}\right) \operatorname{Tr} F_{A}^{2}=\operatorname{Im} \operatorname{tr}\left(\alpha_{1} \alpha_{2}^{t}\right) .
$$

Finally, we shall consider a group action on the moduli spaces which does not arise from an action on $\mathbb{R}^{4}$, namely the $S U(r) / \mathbb{Z}_{r}$ action on the framings of elements of $\mathscr{A}$. This acts on $\mathscr{M}_{k, r}$ with quotient $\mathscr{M}_{k, r}^{u}$ consisting of (unframed) ASD connections modulo the whole gauge group. It acts freely on the irreducible elements $\mathscr{M}_{k, r}^{*}$ of $\mathscr{M}_{k, r}$ and defines a fibration

$$
G / Z(G) \rightarrow \mathscr{M}_{k, r}^{*} \rightarrow \tilde{\mathscr{M}}_{k, r}^{u *}
$$

This is true for any manifold $M$ and for the moment we shall generalize our discussion to the case when $M$ is a Kähler manifold of complex dimension $n$, with base point $x_{0}$, and let $G$ be any compact connected semisimple Lie group. Then it is well known (see [6], pages 237, 238) that there is a moment map for the action of the gauge group $\mathscr{G}$ on the space $\mathscr{A}^{u}$ of (unframed) ASD connections on some $G$-bundle $P$ given by $F_{A} \wedge \omega^{n-1} / \kappa \in \Omega^{2 n}(\operatorname{ad} P) \cong \operatorname{Lie}(\mathscr{G})^{*}$, where $\kappa=2^{3-n} \pi^{2}$.

It is also well known that the space of framed connections modulo gauge can be identified with the space $\mathscr{A}^{\boldsymbol{u}} / \mathscr{G}_{0}$, where $\mathscr{G}_{0}=\left\{\phi \in \mathscr{G} \mid \phi\left(x_{0}\right)=\mathrm{id}\right\}$, and $\mathscr{G} / \mathscr{G}_{0} \cong G$. The centre $Z(G)$ acts trivially on $\mathscr{A}^{u}$ so consider the quotient $H=G / Z(G)$ which acts freely on irreducible elements. We want to find a moment map for the $H$ action on the framings $P_{x_{0}} \cong G$. This can be done by considering the identification $\mathscr{A} / \mathscr{G} \cong \mathscr{A}^{u} / \mathscr{G}_{0}$ as follows.

Fix a basis $\left\{\sigma_{1}, \ldots, \sigma_{\alpha}\right\}$ for $(\operatorname{ad} P)_{x_{0}} \cong \mathfrak{g}$ and extend each element $\sigma_{i}$ to $\tilde{\sigma}_{i}$ by parallel transport along radial lines in a small neighbourhood $N_{\lambda}\left(x_{0}\right)$ of $x_{0}$, by 0 outside $N_{2 \lambda}\left(x_{0}\right)$ and smoothly interpolated in the annulus $\operatorname{Ann}\left(\lambda, 2 \lambda ; x_{0}\right)$ by a bump function. We choose $\lambda$ sufficiently small to allow solutions of $d_{A} \tilde{\sigma}_{i}=0$ to exist along radial lines in $N_{2 \lambda}\left(x_{0}\right)$ such that $\left\{d_{A} \tilde{\sigma}_{i}\right\}$ generates the framing part of $T_{[A]} \mathscr{A} / \mathscr{G}$. This provides us with a model for $T_{e} H=\left\{\tilde{\sigma}_{i}\right\}$, by which we mean an embedding of $T_{e} H$ into $\Omega^{0}(\operatorname{ad} P)$. Using this identification we define $\eta: G \subset \mathscr{G}$ so that the model $\mathfrak{H} \cong T_{e} H \cong T_{e} G \subseteq \Omega^{0}(\operatorname{ad} P) \cong \operatorname{Lie}(\mathscr{G})$ is just $\eta_{*}: T H \rightarrow T \mathscr{G}$. Denote the pullback $\Omega^{2 n}(\operatorname{ad} P) \rightarrow T_{e}^{*} H$ by $\eta^{*}$ and then we can define $\mu: \mathscr{A}^{u} \rightarrow \mathfrak{H}^{*}$ by $\mu(A)=\eta^{*}\left(F_{A} \wedge \omega^{n-1}\right) / \kappa$. This is $H$-equivariant since $F_{A} \wedge \omega^{n-1}$ was $\mathscr{G}$-equivariant and for $\theta \in T_{e} H$ and $\alpha \in \Omega^{1}(\operatorname{ad} P)$ we have

$$
\begin{aligned}
d \mu(\theta)(\alpha) & =\frac{1}{\kappa} \eta^{*}\left[\int \alpha \wedge d_{A}\left(\eta_{*} \theta\right) \wedge \omega^{n-1}\right] \\
& \left.\left.=\eta^{*}\left(d_{A} \eta_{*} \theta\right\lrcorner \Omega\right)(\alpha)=\left(d_{A} \theta\right\lrcorner \Omega\right)(\alpha) .
\end{aligned}
$$

Hence $\mu$ is a moment map for the $H$ action. Now observe that $\mu$ is $\mathscr{G}_{0}$-equivariant and that the coadjoint action of $\mathscr{G}_{0}$ on $\mathfrak{H}^{*}$ is trivial, and so $\mu$ descends to $\mu: \mathscr{A} / \mathscr{G} \rightarrow \mathfrak{H}^{*}$ and hence, by restriction, to $\mu: \mathscr{M} \rightarrow \mathfrak{H}^{*}$, where $\mathscr{M}$ denotes the framed Yang-Mills moduli space. If we use the identification of $\left\{\sigma_{i}\right\}$ with $\left\{\tilde{\sigma}_{i}\right\}$ now in reverse we can identify $\eta^{*}\left(F_{A} \wedge \omega^{n-1}\right)$ with $\left(F_{A} \wedge \omega^{n-1}\right)\left(x_{0}\right) \in \Lambda^{2 n} T_{x_{0}}^{*} M \otimes \mathfrak{S} \cong \mathfrak{H}^{*}$.

We have now proved

Theorem 4.13. Let $(M, \omega)$ be a Kähler manifold of complex dimension $n$ and let $G$ be a compact connected semisimple Lie group. Then a moment map for the action 
of $G / Z(G)$ on the framing of Hermitian-Yang-Mills G-connections modulo gauge and framed at $x \in M$ is given by

$$
\mu([A])=\frac{\eta_{x}^{*}\left(F_{A} \wedge \omega^{n-1}\right)}{2^{3-n} \pi^{2}},
$$

where $\eta_{x}: G \hookrightarrow \mathscr{G}$ is the map defined above so that $\eta_{x}^{*}: \Omega^{2 n}(\operatorname{ad} P) \rightarrow \operatorname{Lie}(G / Z(G))^{*}$ is evaluation at the point $x$.

In the case where $M=\mathbb{R}^{4}$ and we are framing connections at infinity, $x_{0} \notin \mathbb{R}^{4}$ but we can use Uhlenbeck's removability of singularities theorem [15, Cor. 4.3] to make sense of $\left(F_{A} \wedge \omega\right)(\infty)$. Let $s: S^{4} \backslash\{\infty\} \rightarrow \mathbb{R}^{4}$ denote stereographic projection. Then there is a gauge transformation $g \in \mathscr{G}$ such that $\tilde{A}=g(A)$ can be extended across $\infty \in S^{4}$. Moreover, the limit of $\eta_{x}^{*}\left(F_{\tilde{A}} \wedge s_{*} \omega\right)$ as $x \rightarrow \infty$ exists on $S^{4} \backslash\{\infty\}$ and must equal the limit of $\eta_{x}^{*} s^{*}\left(F_{\tilde{A}} \wedge s_{*} \omega\right)$ as $x \rightarrow \infty$ on $\mathbb{R}^{4}$. Hence we have

Corollary 4.14. A moment map for the action of $S U(r) / \mathbb{Z}_{r}$ on $\mathscr{M}_{k, r}$ which acts on the framing of a framed ASD connection $[A]$ is

$$
\mu(a)=\frac{1}{2 \pi^{2}} \lim _{x \rightarrow \infty} \eta_{x}^{*}\left(F_{A} \wedge \omega\right) .
$$

We would like to relate this to the action of $H$ on dual space $\hat{\mathscr{M}}_{r, k}$. Recall that the fibre of $P$ "at infinity" is given by the vector space $L$ in the monad $K \rightarrow K \oplus K \oplus L \rightarrow K$ in the limit $x \rightarrow \infty$. The group $H$ acts on $L$ in the fundamental representation and hence, if we fix $L$, on the matrices $a, b$ by $b \oplus a^{*} \mapsto b y \oplus a^{*} g$, and $H$ fixes $\alpha_{1}$ and $\alpha_{2}$. The moment map $\mu$ for this action can be computed using the fact that $\mu=0$ minimises points of $\hat{\mathscr{M}}_{r, k}$ in a given $H^{c}$ orbit, (see [5], page 458). Using the same calculation with $S U(r)$ instead of $G L(k, \mathbb{C})$ we find $\mu\left(\left[\alpha_{1}, \alpha_{2}, a, b\right]\right)=b^{*} b-a a^{*} \in \mathfrak{s u}(r)$; we identify a Lie algebra with its dual via the Killing form.

As before, the infinitesimal action of $H$ on $\mathscr{M}_{k, r}$ is just the pull-back of the action on $\hat{\mathscr{M}}_{r, k}$ and the metrics agree by Theorem 3.2. Moreover the group $H$ is semisimple and hence moment maps are unique, so we can deduce:

Theorem 4.15. For all $[A] \in \mathscr{M}_{k, r}$ and $\left[\alpha_{1}, \alpha_{2}, a, b\right]=p(A)$,

$$
\frac{1}{2 \pi^{2}} \lim _{x \rightarrow \infty} * \eta_{x}^{*}\left(F_{A} \wedge \omega\right)=b^{*} b-a a^{*} .
$$

We now have explicit formulae for the moment maps and associated functions for the whole symmetry group of $\mathscr{M}_{k, r}$ and $\hat{\mathscr{M}}_{r, k}$.

Acknowledgements. Finally, I would like to thank Simon Donaldson for his unfailing support, Alastair King for many lengthy discussions on this subject and Andrew Swann for drawing my attention to Corollary 4.11 .

\section{References}

1. Atiyah, M. F.: Geometry of Yang -Mills fields. Lez. Ferm. Acc. Naz. dei Lin. Sc. Norm. Sup., Pisa (1979) 
2. - , Hitchin, N. J.: Geometry and dynamics of magnetic monopoles. Princeton, NJ: Princeton University Press 1988

3. Boyer, C. P., Mann, B. M.: A classifying space for instantons. Preprint (1989)

4. Braam, P. J., van Baal, P.: Nahm's transformation for instantons. Commun. Math. Phys. 122, 267-280 (1989)

5. Donaldson, S. K.: Instantons and geometric invariant theory. Commun. Math. Phys. 93, 453-460 (1984)

6. - : Connections, cohomology and the intersection forms of 4-manifolds. J. Diff. Geom. 24, 275-341 (1986)

7. - : Infinite determinants, stable bundles and curvature. Duke Math. J. 54, 231-247 (1987)

8. Freed, D., Uhlenbeck, K.: Instantons and four manifolds. Berlin, Heidelberg, New York: Springer 1984

9. Groisser, D., Parker, T. H.: The Riemannian geometry of the Yang-Mills moduli space. Commun. Math. Phys. 112, 663-689 (1987)

10.,- The geometry of the Yang-Mills moduli space for definite manifolds. J. Diff. Geom. 29, 499-544 (1989)

11. Hitchin, N. J., Karlhede, A., Lindström, U., Roček, M.: Hyper-Kähler metrics and supersymmetry. Commun. Math. Phys. 108, 535-589 (1987)

12. Osborn, H.: Calculation of multi-instanton determinants. Nucl. Phys. B159, 497-511 (1979)

13. Swann, A.: private communication

14. Taubes, C. H.: Stability in Yang-Mills theories. Commun. Math. Phys. 91, 235-263 (1983)

15. Uhlenbeck, K.: Removable singularities in Yang-Mills fields. Commun. Math. Phys. 83, 11-29 (1982)

Communicated by A. Jaffe 